\title{
Characteristic measurements of a wideband gyro- TWA operating in W-band
}

\author{
Wenlong $\mathrm{He}$ \\ College of Electronic Science and \\ Technology \\ Shenzhen University \\ Shenzhen, China \\ wenlong.he@szu.edu.cn
}

Liang Zhang, Craig R. Donaldson, Kevin Ronald, Alan D.R. Phelps and Adrian W. Cross

Department of Physics, SUPA, University of Strathclyde Glasgow, UK

liang.zhang@strath.ac.uk craig.donaldson@strath.ac.uk k.ronald@strath.ac.uk

a.d.r.phelps@strath.ac.uk a.w.cross@strath.ac.uk

\author{
Peter Cain \\ Keysight Technologies UK Ltd \\ Edinburgh, UK \\ pete_cain@yahoo.co.uk
}

\begin{abstract}
Following on from the successful operation of a broadband, high-power gyrotron traveling wave amplifier (gyro-TWA) in W-band, experiments were carried out to demonstrate the versatility of the gyro-TWA and investigate its tuning characteristics. When operating at a lower voltage of 40 $\mathrm{kV}, 30 \mathrm{~dB}$ gain was achieved for a $1 \mathrm{GHz}$ bandwidth frequency-swept input signal.
\end{abstract}

Keywords- gyrotron traveling wave amplifier, broadband amplification, helically corrugated waveguide, frequency-swept

\section{INTRODUCTION}

Gyrotron devices based on the cyclotron resonance maser instability have superior power capability over the slowwave vacuum electron devices. The gyrotron traveling wave amplifier (gyro-TWA) is one of the members of the gyrodevice family and it is able to produce high power coherent microwave radiation at $\mathrm{mm}$-wave and sub-mm-wave frequencies. As an amplifier, the amplitude and phase of the gyro-TWA can be precisely controlled therefore it is attractive in applications such as communications, radar and electron spin resonance spectroscopy.

A W-band gyro-TWA using a helically corrugated waveguide $(\mathrm{HCW})$ as the interaction region has been developed at the University of Strathclyde [1]. The HCW has both azimuthal and axial periodicities which allow to couple two different modes in the circular waveguide to generate new dispersion curves. By carefully choosing the geometry parameters, an ideal dispersion curve that has a nearly constant group velocity at small wavenumber values crossing a large frequency bandwidth can be achieved. The gyro-TWA was driven by an axial encircling large orbit electron beam which was generated by a cusp electron gun. It was able to operate at the $2^{\text {nd }}$ cyclotron harmonic therefore only half of the magnetic field strength as compared to fundamental harmonic interaction was required.

The experiment setup of the gyro-TWA is shown in Fig. 1. The gyro-TWA itself contained key components including cusp electron gun [2, 3], pillbox window [4], input coupler [5], elliptical polarizer, input and output windows [6, 7], HCW, and the solenoid systems. The experiment also required a high-voltage power supply to drive the electron beam.

In previous gyro-TWA experiments, the gain curve was measured at discrete frequency points. Further improvement was achieved by building an integrated signal generation and measurement system using advanced equipment from Keysight Ltd, as shown in Fig. 2. It includes a Keysight arbitrary waveform generator (AWG, model M8190A) to generate arbitrary waveform up to $5 \mathrm{GHz}$ analog bandwidth. The signal was then modulated onto a $\sim 15.5 \mathrm{GHz}$ carrier signal by a Keysight vector signal generator. Then the RF signal was used to drive a low-power solid state source to generate the seed microwave signal for the gyro-TWA. To measure the amplified microwave signal and to determine the power level, a waveguide harmonic mixer (Keysight M1970W), a signal analyzer (Keysight PXA N9030A) and a real-time digitizing oscilloscope (Keysight MSOS804A) were used. All the equipment was remotely controlled.

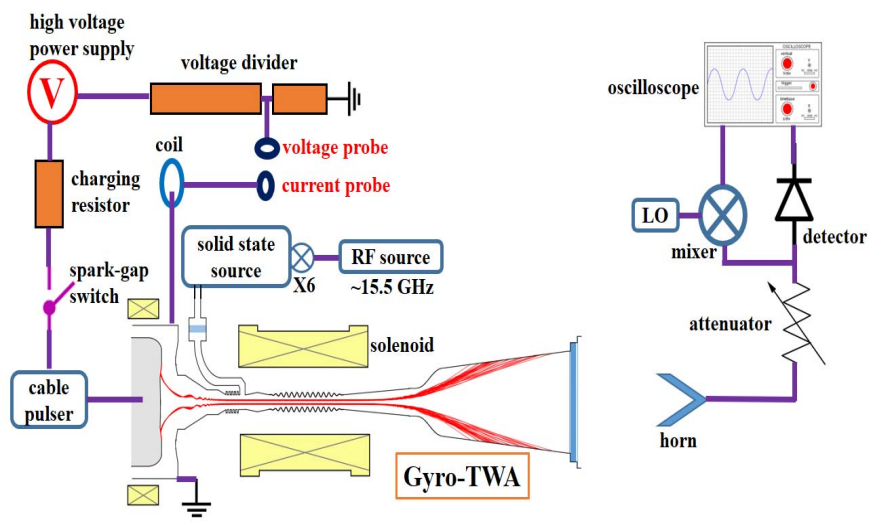

Fig. 1. The experimental setup of the gyro-TWA.

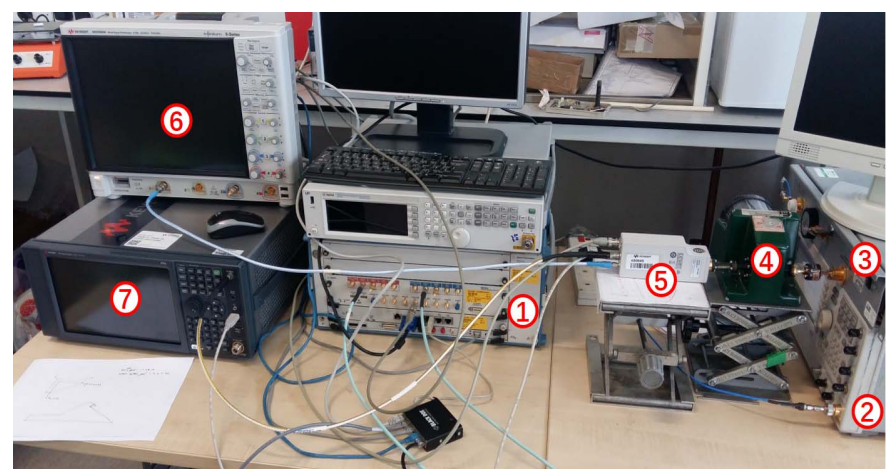

Fig. 2. The setup for generation and measurement of the mm-wave signal

\section{Simulation AND MEASUREMENT RESUltS}

The optimal operating voltage of the gyro-TWA is $55 \mathrm{kV}$. In this experiment, to demonstrate the versatility in 
operating the gyro-TWA the operating beam voltage was lowered to $40 \mathrm{kV}$. In this case, the other parameters, such as the beam alpha value and the magnetic field strength were needed to be adjusted accordingly. Fig. 3 shows the particlein-cell (PIC) simulations with the new parameter sets using the 3D PIC code MAGIC. As compared with higher operating voltage, the gain dropped about $7 \mathrm{~dB}$ and the bandwidth becomes smaller. However, the radiated microwave power level was still more than $1 \mathrm{~kW}$.

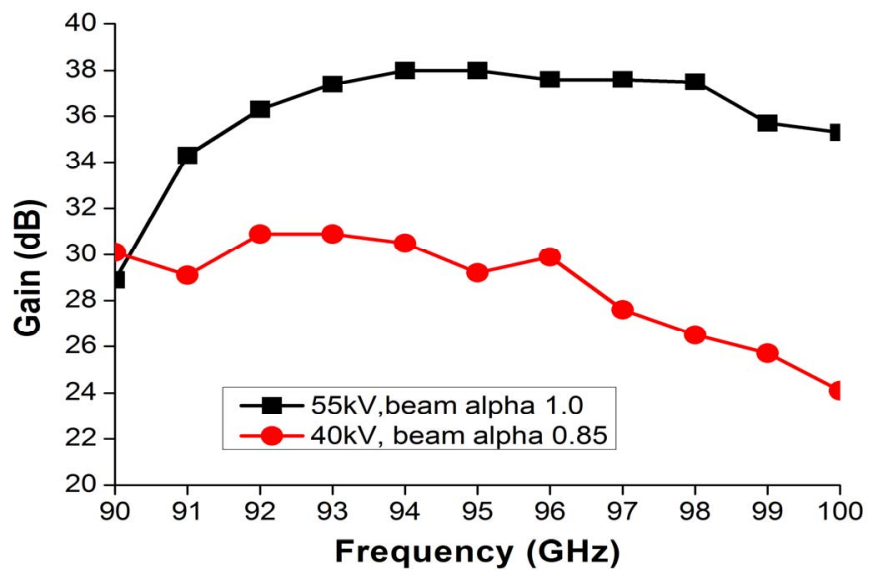

Fig. 3. Comparison of the gain at different parameter sets.

In the experiment, it was found that it is difficult to achieve a desired input signal beyond the bandwidth of 1 $\mathrm{GHz}$. Therefore the frequency-swept signal was set to a bandwidth of $1.0 \mathrm{GHz}$ at the central frequency of $93.5 \mathrm{GHz}$. From Fig. 4(a), the noise of the frequency-swept signal generated from the solid-state source was relatively large due to the nonlinear behavior of the frequency multipliers. The amplified signal in Fig. 4(a) showed clear frequency sweeping that aligned well with the input signal. The correlated mm-wave signal and the beam voltage is shown in Fig. 4(b). The spectrum of the amplified mm-wave signal in Fig. 4(c) showed a bandwidth close but smaller than 1 $\mathrm{GHz}$. The phase information after post-processing shows good agreement between the input and amplified signals [8].

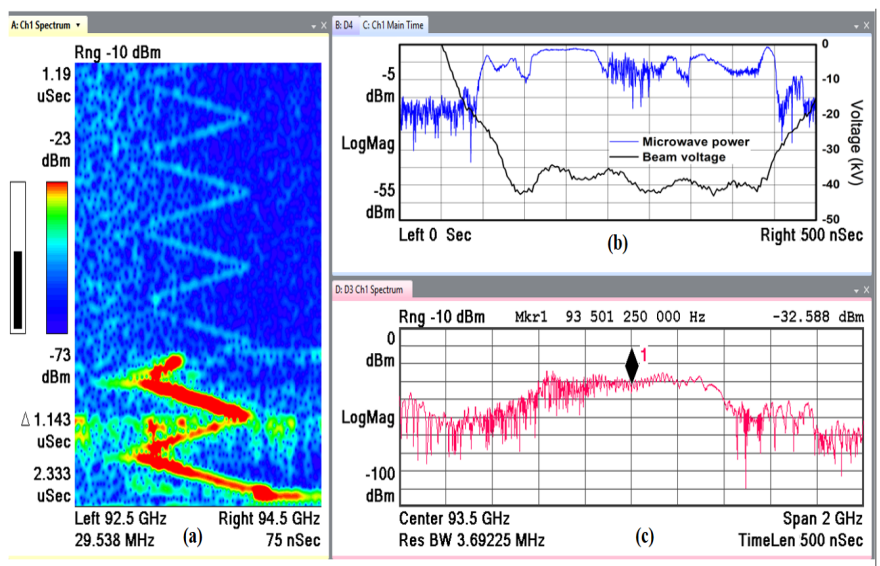

Fig. 4. Example Gyro-TWA experimental results with the frequencyswept signal. (a) spectrogram, (b) correlation plot of the mm-wave signal and the acceleration voltage, and (c) spectrum of the mm-wave signal.

\section{CONCLUSION}

Frequency-swept experiments were carried out on a Wband gyro-TWA to investigate its characteristics on broadband amplification and its gains at different operating voltages. A $30 \mathrm{~dB}$ gain was achieved when it was driven by a $40 \mathrm{kV}, 1.5 \mathrm{~A}$ electron beam. The measured results were in excellent agreement with the simulation prediction. The verification of the frequency agility of the W-band gyroTWA enabled the possibility to carry out experiments for applications in the area of radar, communications and electron spin resonance spectroscopy.

The wider bandwidth experiment was limited by the nonlinear behavior from the frequency multipliers in the solid-state source. The future improvements of the gyroTWA include sourcing a wider bandwidth solid-state source as well as a more stable high-voltage power supply to drive the electron beam.

\section{ACKNOWLEDGMENT}

This work was supported by EPSRC UK (research Grant No. EP/G036659/1, EP/K029746/1) and STFC UK (research Grant No. ST/N002326/1 and ST/P001890/1).

\section{REFERENCES}

[1] W. He, C. R. Donaldson, L. Zhang, K. Ronald, A. D. R. Phelps, and A. W. Cross, "Broadband Amplification of Low-Terahertz Signals Using Axis-Encircling Electrons in a Helically Corrugated Interaction Region," Phys. Rev. Lett., vol. 119, no. 18, p. 184801, Oct. 2017. DOI: 10.1103/PhysRevLett.119.184801.

[2] C. R. Donaldson, W. He, A. W. Cross, F. Li, A. D. R. Phelps, L. Zhang, K. Ronald, C. W. Robertson, C. G. Whyte, and A. R. Young, "A cusp electron gun for millimeter wave gyrodevices," Appl. Phys. Lett., vol. 96, no. 14, p. 141501, 2010. DOI: 10.1063/1.3374888.

[3] L. Zhang, W. He, C. R. Donaldson, and A. W. Cross, "Investigation on the optimal magnetic field of a cusp electron gun for a W-band gyro-TWA," Physics of Plasmas, vol. 25, no. 5, p. 053104, 2018/05/01 2018. DOI: 10.1063/1.5027070.

[4] L. Zhang, C. R. Donaldson, A. W. Cross, and W. He, "A pillbox window with impedance matching sections for a W-band gyroTWA," IEEE Electron Device Letters, pp. 1-1, 2018. DOI: 10.1109/LED.2018.2834859.

[5] L. Zhang, W. He, C. R. Donaldson, J. R. Garner, P. McElhinney, and A. W. Cross, "Design and Measurement of a Broadband Sidewall Coupler for a W-Band Gyro-TWA," IEEE Trans. Microwave Theory Techn., vol. 63, no. 10, pp. 3183-3190, Oct. 2015. DOI: 10.1109/TMTT.2015.2464302.

[6] L. Zhang, W. He, C. R. Donaldson, G. M. Smith, D. A. Robertson, R. I. Hunter, and A. W. Cross, "Optimization and Measurement of a Smoothly Profiled Horn for a W-Band Gyro-TWA," IEEE Trans. Electron Devices, vol. 64, no. 6, pp. 2665-2669, June 2017. DOI: 10.1109/TED.2017.2687949.

[7] C. R. Donaldson, P. McElhinney, L. Zhang, and W. He, "Wide-Band HE11 Mode Terahertz Wave Windows for Gyro-Amplifiers," IEEE Transactions on Terahertz Science and Technology, vol. 6, no. 1, pp. 108-112, June 2016. DOI: 10.1109/TTHZ.2015.2495221.

[8] L. Zhang, C. R. Donaldson, P. Cain, A. W. Cross, and W. He, "Amplification of frequency-swept signals in a W-band gyrotron travelling wave amplifier," IEEE Electron Device Letters, pp. 1-1, 2018. DOI: 10.1109/LED.2018.2836868. 